combined traumas were in similar proportions.

The children with the crush syndrome were the most severely compromised patients. Most often, the crush injury was localised to the ankle (30\%), thigh (28.6\%), head $(23.8 \%)$, or forearm (7\%). Of all children with crush syndrome, $12.6 \%$ required amputations. Positive results were seen in children who had fasciotomy with early plastic surgery procedures to the skin. Acute renal insufficiency has been reported in $27 \%$ of children with crush syndrome.

Despite generally positive results of treating child victims, we could have had even better results if all children had been concentrated in one hospital, and had had, from the beginning, qualified pediatric help.

Key words: amputations; children; crush syndrome; earthquake; injuries; pediatricians

Prehosp Disast Med 2001;16(2):s63.

\section{Principles of Rendering Medical Aid to Children in Disasters}

Professor Leonid. M. Roshal, MD, PbD, DSc. (Russia)

Chairman, International Committee on Pediatric Disasters and Wars, World Association of Disaster and Emergency Mecdicine (WADEM)

Our experience in rendering medical aid to children in emergency situations in many countries of the world (Afghanistan, Armenia, Egypt, Georgia, India, Iran, Japan, Russia, Turkey) has enabled us to formulate some principles that should help to decrease mortality and morbidity rates among injured children:

1. Medical aid should be rendered by pediatric specialists

2. Specialized medical aid in case of disaster should be as close to the site of disaster as possible

3. Pediatric intensive care specialists and pediatriciantraumatologists, as well as physicians who have had special training courses on rendering medical assistance to children in extreme situations and having a proper license should work in the disaster areas

4. Transportation of an injured child to a hospital should be done only if the child is transportable, and only after all preliminary intensive care measures are performed at the site

5. Children from a disaster area must be concentrated in one, or at most, two hospitals. The time of transportation, if all necessary curative measures are performed simultaneously, is of no importance. It is important for the child to be hospitalized at a specialized pediatric hospital that has many types of pediatric specialists on emergency therapy, traumatology, plastic surgery, pediatric surgery, and nephrology. This hospital should be equipped with a modern laboratory and other equipment like a computerized tomographic scanner, renal dialyzer, etc.

Key words: children; credentials; disasters; intensive care; pediatricians; principles; specialization; transportation; treatment

Prebosp Disast Med 2001;16(2):s63.
SWEDE: A Management System with Internet Technology Support for the Health Care System in Emergency and Disaster Situations Anders Rüter, $M D$

Swedish National Board of Health and Welfare, and the Centre for Teaching and Research in Disaster Medicine, University Hospital, Linköping, SWEDEN

The Swedish National Board of Health and Welfare has introduced SWEDE, a new management system to ensure a high level of preparedness for major emergencies and disaster situations. The SWEDE system is based on a general protocol and a computerised information system-IS Swede. The protocol standardises terminology, enabling emergency personnel to cooperate and coordinate activities during major emergencies and disasters in a more efficient way. The protocol is understood and accepted by the health care system, and is similar to those used by police and rescue services. The information system includes patient information as well as information on available resources.

New equipment has been introduced in ambulances. Information is sent on-line to receiving hospitals using the Mobitex ${ }^{\circledR}$ system and Internet technology. In situations of disaster or during a state of alert, this system provides continuous access to relevant information.

One important part of the SWEDE concept is that it is used daily and, when situations escalate, it is already in place and can be mobilized rapidly. Where introduced, the IS Swede today is used routinely in all situations involving an ambulance. The information is sent to a central database then directed to the receiving hospital by encrypted Internet. The hospital receives advance information about the accident, the patient(s), and the treatment being given at scene and during transport. In disaster situations, the management group can also use the IS Swede to direct the ambulances to those hospitals having the necessary resources.

Currently, the system has been introduced in four county councils in Sweden. Other county councils, as well as the Swedish National Defence, are considering the system and it is proposed that $75 \%$ of all county councils in Sweden should have the SWEDE system in use at the end of 2005. Key words: ambulances; disaster; emergencies; information systems; Internet; management systems; structure; Sweden; terminology

E-mail: anders.ruter@lio.se

Prehosp Disast Med 2001;16(2):s63.

\section{Amendment in the Regulations of the Emergency Plan ("Red Plan") in Case of Major Accidents with Limited Consequences in a City \\ M. Rüttimann; B. Tabuteau; J-J. Kowalski}

Emergency Medical Service, Paris Firefighter Brigade, Paris, FRANCE

The "Red Plan" is a one of several emergency plans destined to help numerous victims. A decree details the circumstances in which the plan can be launched and just how it should develop at a regional or "department" level 